International Journal of Quantum Information

(C) World Scientific Publishing Company

\title{
Paramagnetic Materials and Practical Algorithmic Cooling for NMR Quantum Computing*
}

\author{
JOSÉ M. FERNANDEZ \\ Départment de génie informatique, École Polytechnique \\ Montréal (Quebéc) Canada \\ jose.fernandez@polymtl.ca \\ TAL MOR and YOSSI WEINSTEIN \\ Faculty of Computer Science \\ Technion - Israel Institute of Technology \\ Haifa, Israel \\ talmo@cs.technion.ac.il andyossiv@cs.technion.ac.il
}

Received (1 July 2004)

\begin{abstract}
Algorithmic Cooling is a method that uses novel data compression techniques and simple quantum computing devices to improve NMR spectroscopy, and to offer scalable NMR quantum computers. The algorithm recursively employs two steps. A reversible entropy compression of the computation quantum-bits (qubits) of the system and an irreversible heat transfer from the system to the environment through a set of reset qubits that reach thermal relaxation rapidly.

Is it possible to experimentally demonstrate algorithmic cooling using existing technology? To allow experimental algorithmic cooling, the thermalization time of the reset qubits must be much shorter than the thermalization time of the computation qubits. However such thermalization-times ratios have yet to be reported.

We investigate here the effect of a paramagnetic salt on the thermalization-times ratio of computation qubits (carbons) and a reset qubit (hydrogen). We show that the thermalization-times ratio is improved by approximately three-fold. Based on this result, an experimental demonstration of algorithmic cooling by thermalization and magnetic
\end{abstract} ions is currently performed by our group and collaborators.

Keywords: Algorithmic Cooling; spin cooling; Thermalization times ratio; paramagnetic salt.

\section{Introduction}

NMR Quantum Computing (NMRQC) was proposed in 1997 by Cory, Fahmy and Havel $^{1}$, and by Chuang and Gershenfeld ${ }^{2}$. They succeeded in implementing quantum algorithms on four-state quantum systems (two spins). They used a liquid, an ensemble of identical molecules. For encoding a quantum bit (qubit), the nuclear

*A conference version of this paper appeared in SPIE, volume 5105, pages 185-194 (2003) 
spin of one of the atoms in the molecule is used. Each molecule is an independent quantum computer. When measuring a qubit we get an average of the values of that qubit over the ensemble. This technique is called ensemble quantum computing, and in order to achieve quantum computation using this method, modifications were introduced into the algorithms since they were developed for a single quantum computer $^{2,3}$. To this day liquid NMR is the most successful way of doing quantum computation. NMR spectroscopists have succeeded in performing computation with up to seven qubits in liquid $\mathrm{NMR}^{4,5}$, while other successful methods have barely reached two-four qubits, and many promising solid-state NMR methods are still struggling to encode a single qubit.

The probability of a spin-half which is coupled to a thermal bath and a constant magnetic field to be in the states $|\uparrow\rangle$ and $|\downarrow\rangle$ are given by $P_{\uparrow}=(1+\varepsilon) / 2, P_{\downarrow}=$ $(1-\varepsilon) / 2$. The population bias $\varepsilon$ is defined as $\varepsilon \triangleq \tanh [(\Delta E) /(2 K T)]$, where $\Delta E$ is the energy gap between the two spin states, $K$ is the Boltzman coefficient and $T$ is the temperature in Kelvin.

Let each spin $\frac{1}{2}$ particle be considered as a qubit. In a string of qubits (e.g. a molecule composed of several atoms with nuclear spin $\frac{1}{2}$ ) the thermal state density matrix of the $\mathrm{i}^{\text {th }}$ qubit is

$$
\rho_{\varepsilon_{0}^{i}}=\frac{1}{2}\left(\begin{array}{cc}
1+\varepsilon^{i} & 0 \\
0 & 1-\varepsilon^{i}
\end{array}\right) .
$$

At room temperature the largest $\varepsilon$ reached so far is $\varepsilon \sim 10^{-5}$. $\varepsilon$ is also known as the polarization bias, since it tells us the tendency of the system to prefer one state over the other. The signal received from the sample originates from this bias; the larger the bias, the stronger the signal. The state of the molecule is described by a tensor product of the single-qubit states, $\rho=\rho_{\varepsilon_{0}^{1}} \otimes \cdots \otimes \rho_{\varepsilon_{0}^{n}}$ for $n$ qubits.

Unfortunately, the NMR quantum computing technique cannot work directly with such mixed states. The algorithms are modified to use a pseudo-pure state and then suffer from inherently bad signal-to-noise ratio (SNR). The SNR then decreases exponentially as the number of qubits grows $6,7,8,9,10$.

\section{Algorithmic Cooling of Spins}

The scaling problem of NMR quantum computers can be resolved by pre-cooling the qubits, which increases their bias, via two novel techniques recently developed. First, an adiabatic (reversible) cooling scheme has been introduced ${ }^{8}$, which solves the scaling problem using data compression tools (that can actually be viewed as polarization compression tools). This scheme does not suffer from the SNR problem anymore, but is bounded by Shannon's bound on entropy-preserving compression, and therefore is limited in its practicality. Later on, Algorithmic Cooling ${ }^{9}$, a novel polarization compression technique, was suggested. It combines reversible compression together with thermalization steps; by opening the system to the environment, and using thermalization (!!) as a cooling mechanism, Shannon's bound is bypassed. 
An efficient and experimentally feasible algorithmic cooling was then suggested ${ }^{10}$, with potentially important applications already in the near future: improving SNR in NMR spectroscopy due to effective cooling of spins. As far as we know, that work ${ }^{10}$ provided the first near-future application of quantum computing devices.

One can improve the SNR by cooling the system, increasing the magnetic field, or increasing the sample size or number of sampling steps. Another solution (that has some advantages over the above ${ }^{10}$ and can be combined with these other strategies as well) is an effective cooling of spins. This is a way to increase the spin bias without cooling the system nor increasing the magnetic field. Such a "spincooling" technique means taking the spin out of thermal equilibrium, then using the improved polarization for spectroscopy before the spin goes back to its thermal equilibrium state. A well known example of such a spin-cooling technique is the polarization transfer between two spins that have different polarization biases (e.g., proton and carbon spins). Doing this, the proton is heated four times while the carbon is cooled accordingly.

The reversible (in-place) polarization compression ${ }^{8}$ mentioned before is another spin-cooling technique. It compresses the entropy from a few nuclear spins on a molecule to other spins on the same molecule. Similar to the polarization transfer, this cooling method is also reversible, and therefore it preserves the entropy contained in the system. It is limited due to the Shannon's bound: in order to have $n_{j}$ pure-state qubits we would need to start with $n_{0}=\ln (4) n_{j} \varepsilon_{0}^{-2}$ qubits ${ }^{8}$.

In contrast, algorithmic cooling uses "irreversible" steps as well. In its simplest form, each qubit is assigned a neighboring reset qubit to which the entropy is compressed. The reset qubits thermalize rapidly by radiating the compressed entropy out to the environment, therefore losing heat and letting the entire spin system get cooler. Using a recursive algorithm based on these three steps, adiabatic compression, polarization transfer, and thermalization, Shannon's bound can be bypassed $^{9,10}$ (by far), and low spin temperatures can be reached.

\section{Increasing Thermalization Times Ratio}

Polarization transfer is common in experimental NMR spectroscopy. Furthermore, an experimental demonstration of a reversible polarization compression has already been performed ${ }^{11}$ on molecules of $\mathrm{C}_{2} \mathrm{~F}_{3} \mathrm{Br}$. Thus, the main limitation, preventing the demonstration of algorithmic cooling and the bypassing of Shannon's bound, is that the thermalization-times ratio between the computer qubits and the reset qubits should be large enough so that the cooled computer qubits will not reheat while the reset qubits radiate the compressed entropy. We present and demonstrate now a novel experimental technique to improve the thermalization-times ratio in a real molecule, opening the door for the first algorithmic cooling experiment.

NMR pulse sequences are of typical time duration of milliseconds, while the typical spin thermalization times in liquids are seconds. Hence most of the sequence is spent on waiting for the reset qubits to thermalize. When a physical system is 
taken out of equilibrium, the relaxation process back to equilibrium is always active. So not only do the warm reset qubits cool down, but also the cooled spins warm back up. This warming process is undesired, and therefore we need to find a way to minimize it. If we succeed in increasing the $T_{1}$ ratio between the computational qubits and the reset qubits, the cooled qubits thermalization would play a smaller rule during the reset qubits thermalization.

The use of paramagnetic salts for reducing the thermalization times is a common practice in NMR spectroscopy. Can it also be used to improve the thermalizationtimes ratio? If we use protons as reset qubits and carbons as computation qubits, it may well be that a paramagnetic salt will increase the $T_{1}$ ratio. A good reason to believe so is that, in a typical carbon-chain molecule, the protons are more exposed than the carbons to the environment solution.

We took trichloroethylene (TCE) with two ${ }^{13} \mathrm{C}$ nuclei (spin $1 / 2$ ) used as computation qubits, and one proton (spin $1 / 2$ as well) used as a reset qubit. The TCE was dissolved in deuterated chloroform. We added the salt chromium(III)acetylacetonate (AKA chromium 2,4-pentanedionate) to the solution at a concentration of $233.2 \mathrm{mg} /$ liter. Due to the physical structure of the TCE molecule, the proton has a significantly stronger contact with the magnetic ions than the carbons. The strong contact with the ions indeed decreases the thermalization time, $T_{1}$, of the proton significantly compared to the carbons, achieving the goal of increasing the ratio of the $T_{1}$ 's. This is a very strong effect which can be observed by adding as little salt as we did. Naturally, the dephasing time, $T_{2}$, of all spins, also decreases. $T_{2}$ sets a boundary on the time available for computing. This is since any quantum information, which was obtained in the molecule, is lost due to dephasing. Therefore the ionic salt concentration should allow enough time to perform a computation.

We used a BRUKER DMX-600MHz spectrometer at the University of Montreal. The thermalization time (see Table 1 ) of the proton decreased by $65.6 \%$ while the

Table 1. Thermal relaxation times in TCE before and after adding chromium salt to the solvent.

\begin{tabular}{lll}
\hline Label & unsalted & salted \\
\hline$T_{1}(C 2)$ & $30.85 \mathrm{sec}$ & $28.3 \mathrm{sec}$ \\
$T_{1}(C 1)$ & $27.45 \mathrm{sec}$ & $16.0 \mathrm{sec}$ \\
$T_{1}(H)$ & $5.460 \mathrm{sec}$ & $1.88 \mathrm{sec}$ \\
$T_{1}(C 2) / T_{1}(H)$ & 5.65 & 15.05 \\
$T_{1}(C 1) / T_{1}(H)$ & 5.03 & 8.51 \\
\hline
\end{tabular}

nearest neighbor of the proton, $\mathrm{C} 1$, and the next to nearest neighbor, $\mathrm{C} 2$, changed their thermalization times by $41.7 \%$ and $8.3 \%$ respectively. Meaning that the ratio between the carbons and proton thermalization times went up by $69.2 \%$ for $\mathrm{C} 1$ and by $166.4 \%$ for $\mathrm{C} 2$. These ratios enable an experiment ${ }^{12}$ that can bypass Shannon's 
bound via some form of algorithmic cooling, in which two thermalization steps are performed.

\section{Conclusions}

We showed here that paramagnetic materials can be used to increase the ratio of thermalization times of two spins on a molecule. This effect is most useful for algorithmic cooling, a novel process that improves signal-to-noise ratio in NMR spectroscopy, offering a solution to the scalability problem of NMR quantum computers. We greatly improved the ratio of the thermalization times of carbons and hydrogen, in trichloroethylene molecules, enabling their use for algorithmic cooling. Based on the results presented here, the first experiment presenting some form of algorithmic cooling is currently being performed ${ }^{12}$.

Algorithmic Cooling has a patent pending No. 60/389,208.

\section{Acknowledgments}

This work was supported in parts by the Israeli MOD and the Institute for Future Defense Research. We thank Yuval Elias and Matty Katz for helpful discussions.

\section{References}

1. D. G. Cory and A. F. Fahmy and T. F. Havel, "Ensemble Quantum Computing by Nuclear Magnetic Resonance Spectroscopy", Proc. Natl. Acad. Sci. USA 94, 1634 (1997).

2. N. A. Gershenfeld and I. L. Chuang, "Bulk Spin-Resonance Quantum Computation", Science 275, 350 (1997).

3. P. O. Boykin and T. Mor and V. Roychowdhury and F. Vatan, "Algorithms on Ensemble Quantum Computers", quant-ph/9907067, (1999)

4. L. M. K. Vandersypen and M. Steffen and G. Breyta and C. S. Yannoni and M. H. Sherwood and I. L. Chuang "Experimental Realization of Shor's Quantum Factoring Algorithm Using Nuclear Magnetic Resonance", , Nature 414, 883 (2001).

5. D. Wei and X. Yang and J. Luo and X. Sun and X. Zeng and M. Liu and S. Ding, "Experimental Realization of 7-Qubit Universal Perfect Controlled-NOT and Controlled Square-Root NOT Gates", quant-ph/0109002, (2001).

6. W. S. Warren, "The Usefulness of NMR Quantum Computing", Nature 277, 1688 (1997).

7. D. P. DiVincenzo, "Real and Realistic Quantum Computers", Nature 393, 113 (1998).

8. L. J. Schulman and U. Vazirani, "Molecular Scale Heat Engines and Scalable Quantum Computation", STOC'99, 322 (1999).

9. P. O. Boykin and T. Mor and V. Roychowdhury and F. Vatan and R. Vrijen, "Algorithmic Cooling and Scalable NMR Quantum Computing", Natl. Acad. Sci. USA 99, 3388 (2002).

10. J. M. Fernandez and S. Lloyd and T. Mor and V. Roychowdhury, "Efficient and Experimentally Feasible Algorithmic Cooling", quant-ph/0401135, (2004).

11. D. E. Chang and L. M. K. Vandersypen and M. Stefen, "NMR implementation of a building block for scalable quantum computation", Chem. Phys. Lett. 338, 337 (2001).

12. G. Brassard and R. Laflamme and J. M. Fernandez and T. Mor and Y. Weinstein, "Improved Polarization Transfer with Environment Thermalization", In preparation. 